\title{
«Fürsorge ist der Kerngedanke der Medizin»
}

\author{
Interview: Bruno Kesseli \\ Dr. med. et lic. phil., Chefredaktor der SÄZ
}

«Patientenwohl und Ökonomie - Ärzte als Diener zweier Herren» lautete der Titel eines Referats, das Nikola Biller-Andorno im vergangenen Herbst vor den Delegierten der Ärztekammer hielt. Daran anknüpfend, werden im folgenden Interview einige Fragen dieser für die Ärzteschaft hochaktuellen und spannungsgeladenen Thematik aufgegriffen.

Es besteht ein breiter Konsens darüber, dass die Schweiz eines der besten Gesundheitssysteme der Welt hat. Dass eine Versorgung auf diesem Niveau etwas kostet, ist klar. Wo liegt also das Problem?

«Es ist zwar ein bisschen teuer, aber Qualität kostet halt»: Auf einer solchen Aussage kann man sich ganz gut ausruhen. Die Tatsache, dass unser Gesundheitssystem im Prinzip gut funktioniert, bringt die Versuchung mit sich, kritische Punkte auszublenden, von denen wir wissen, dass es sie gibt.

\section{Zum Beispiel?}

Man kann dazu eine ganze Reihe von Fragen stellen. Wenn wir schon so viel investieren, kriegen wir dann auch die entsprechende Leistung aus dem System heraus? Haben wir verlässliche Daten dazu, wie gut, wie fair und effizient unser System ist? Besteht Transparenz bezüglich dieser Daten? Können wir uns dieses System nachhaltig leisten, das heisst, können wir davon ausgehen, dass unsere Kinder, unsere Enkel ebenfalls von einem qualitativ hochwertigen System profitieren werden, das für alle zugänglich ist? Wer sich solche Fragen stellt, kann die Kosten nicht ausblenden, sondern muss sich damit befassen.

\section{Ist das Sache der Ärztinnen und Ärzte?}

Natürlich müssen sich Ärztinnen und Ärzte primär um das Patientenwohl kümmern. Auf der anderen Seite sollten sie aber auch dazu beitragen, dass die Kosten nicht aus dem Ruder laufen. Das kann natürlich zu Konflikten führen.

Sie haben den Grundkonflikt bei Ihrem Referat vor der Ärztekammer mit "Diener zweier Herren» bereits im Titel angedeutet. Wo sehen Sie denn besonders gefährdete oder sensible Bereiche?

Prof. Dr. med. Dr. phil. Nikola Biller-Andorno ist Direktorin des Instituts für Biomedizinische Ethik und Medizingeschichte sowie Leiterin des neugegründeten Center for Medical Humanities der Universität Zürich.

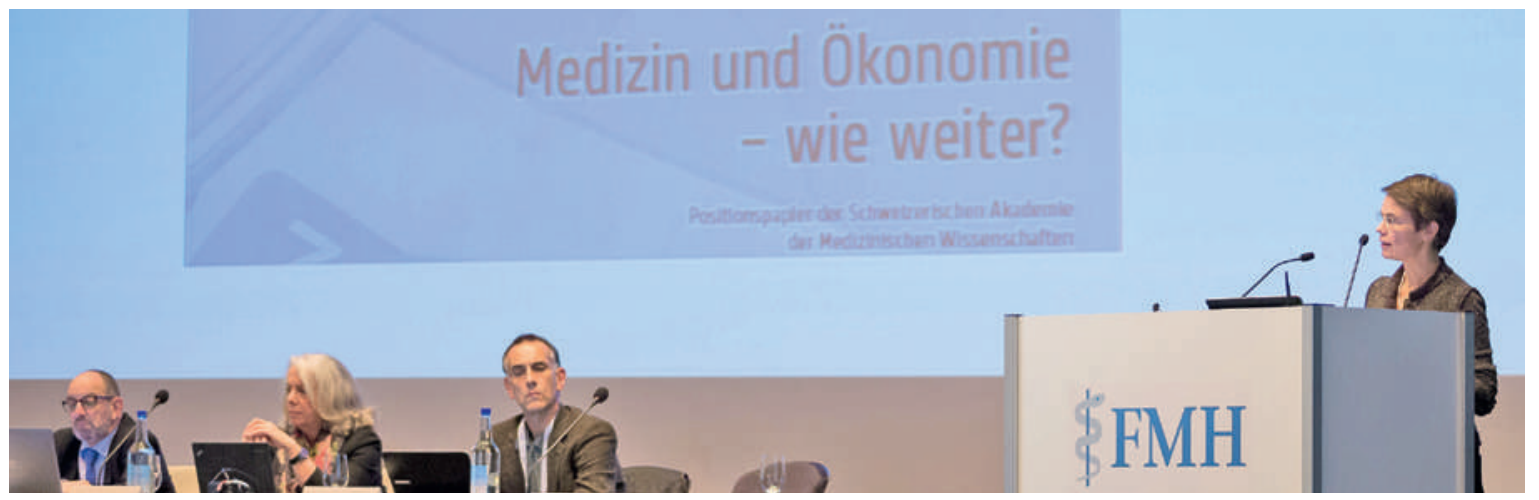

Keine «faulen Kompromisse» eingehen, aber die Kostenfrage nicht ausblenden: Nikola Biller-Andorno bei ihrem Referat vor der Ärztekammer in Biel im Oktober 2014. 
In meinem Referat beziehe ich mich auf das Positionspapier der SAMW [1], das die wichtigsten Problemfelder beschreibt und dabei Fallvignetten zur Illustration anführt. Wenn beispielsweise eine Pflegefachperson nicht mehr dazu kommt, einen Patienten zu mobilisieren oder eine Patientin umzulagern, haben wir damit zum einen eine Qualitätseinbusse durch eine fehlgeleitete Effizienzsteigerung. Zugleich empfindet die Pflegende auch selbst, dass sie nicht die Fürsorge geben kann, die sie gerne geben möchte.

\section{«Es kann Qualitätseinbussen geben,} die mit unseren Standardqualitätsparametern nicht erfasst werden.»

\section{Welchen Stellenwert messen Sie der Fürsorge bei?}

Fürsorge ist der Kerngedanke der Medizin: Ich wende mich jemand anderem zu. Wenn dieser Gedanke durch veränderte Arbeitsbedingungen ausgehöhlt wird, kann die intrinsische Motivation verloren gehen. So wie bei der Pflegenden aus dem eben erwähnten Beispiel, die sagt: «Ich habe diesen Beruf vor Jahren mit Freude gelernt, und jetzt bin ich so weit, dass ich die Patientin einfach im Bett liegen lasse.» Es kann viel verlorengehen, wenn man in einer ungeschickten Weise versucht, Effizienz zu steigern oder Profit zu maximieren.

In ersten Bilanzierungen war zu lesen, dass sich durch DRG in den Spitälern nicht viel geändert habe. In Ihren Fallbeispielen zeichnen Sie ein anderes Bild. Wie kommt das?

Es kommt darauf an, wo man hinschaut. Es gibt zum Beispiel Studien, die zeigen, dass sich an der Verweildauer der Patienten im Spital nicht viel geändert hat. Die Frage ist, ob man sich mit solchen Aussagen zufrieden gibt. Man darf daraus nicht den Schluss ziehen, dass qualitätsmässig nichts passiert. Es kann Qualitätseinbussen geben, die mit unseren Standardqualitätsparametern nicht erfasst werden.

\section{Wie können solche Veränderungen denn erfasst werden?}

Das Bild muss ergänzt werden durch empirische Studien, die auf die Wahrnehmung von denjenigen abstellen, die im Gesundheitswesen wirklich tätig sind, also Ärzte, Pflegende oder auch Angehörige anderer Gesundheitsberufe. Diesen Ansatz haben wir in einer Studie im Rahmen des vom SNF geförderten IDoCProjekts* [2] verfolgt. Ein Subprojekt bestand in einer Umfrage mit stationär-klinisch tätigen Ärztinnen und Ärzten aus verschiedenen Disziplinen, darunter
Innere Medizin, Chirurgie und Anästhesie. Auch die verschiedenen Hierarchiestufen waren gut repräsentiert.

\section{Welche Fragestellungen haben Sie in diesem Subprojekt untersucht?}

Im Zentrum stand die Frage, ob bei der Behandlung der Patienten in der Wahrnehmung der Ärzte andere Entscheide getroffen werden, als man treffen würde, wenn man allein am Patientenwohl orientiert wäre. Es ging also darum, ob ökonomische Erwägungen auf medizinische Behandlungen Einfluss nehmen und ob darunter die Versorgungsqualität und -gerechtigkeit leiden.

\section{Und was ist dabei herausgekommen?}

Interessanterweise wird die Versorgungsqualität in der eigenen Abteilung überwiegend als gut oder sogar sehr gut wahrgenommen. Zugleich wird aber festgestellt, dass sich durchaus Konstellationen ergeben haben, die die Versorgungsqualität beeinträchtigen, durch zu ökonomisch motivierte, zu frühe oder zu späte Entlassungen oder durch verschiedene Formen der Unter- und Überversorgung. Zudem wird seit der Einführung der DRG eher eine Verschlechterung der Arbeitszufriedenheit konstatiert.

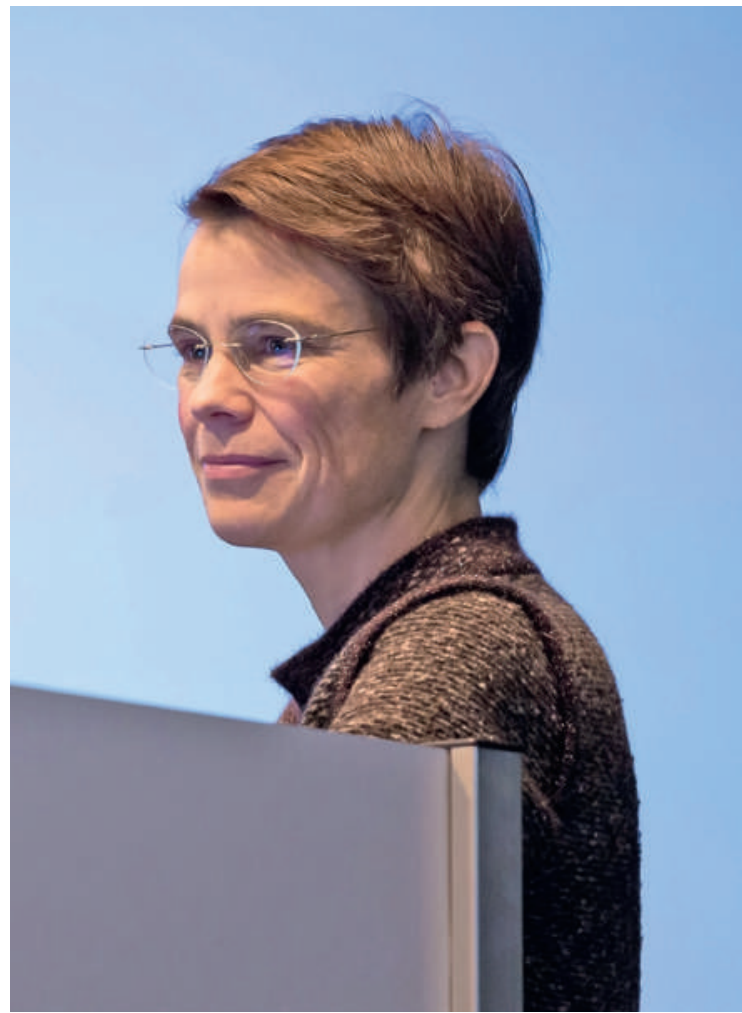

«Es kann viel verlorengehen»: Nikola Biller-Andorno über fehlgeleitete Effizienzsteigerung. 
Können Sie die Art der Befragung an einem konkreten Beispiel verdeutlichen?

Wir haben zum Beispiel gefragt, ob die Studienteilnehmer in ihrem Arbeitsumfeld in den vergangen sechs Monaten erlebt haben, dass eine Massnahme, die für den Patienten nützlich gewesen wäre, aus Kostengründen nicht durchgeführt oder durch etwas Billigeres und weniger Wirksames ersetzt worden ist.

\section{Mit welchem Ergebnis?}

Ich hätte erwartet, dass aufgrund der guten Ressourcenlage in der Schweiz die grosse Mehrheit antwortet: «Das sehen wir niemals.» Aber diese Antwort erhielten wir nur von rund einem Drittel der Befragten. Zwei Drittel sagten: «Das sehen wir», wenngleich mit unterschiedlicher Frequenz. Das sind schon alarmierende Befunde.

\section{Was lässt sich daraus schliessen?}

Diese Art Umfrage reicht für sich genommen natürlich nicht aus, um genau zu erklären, wie sich die medizinische Versorgung entwickelt. Aber die Resultate sind sicherlich ein deutlicher Hinweis darauf, dass man genauer hinschauen muss. Wie kommt es, dass uns ein hoher Prozentsatz der Ärzte sagt: «Wir betreiben bisweilen Unterversorgung»?

\section{Gibt es auch die gegenteilige Tendenz?}

Ja, die gibt es tatsächlich. Wir haben auch gefragt, ob medizinische Interventionen aus ökonomischen Gründen durchgeführt werden, obwohl sie nicht not-

\section{"Ärztinnen und Ärzte handeln also öko- nomischer, als sie dies eigentlich gerne täten.»}

wendig sind. Und auch da sagte knapp die Hälfte der Befragten, dass sie solche Interventionen in den letzten sechs Monaten gesehen haben.

\section{Lässt sich überhaupt schlüssig beurteilen, ob sich die Situation durch die Einführung der DRG verändert hat? Oder war das vielleicht schon vorher so?}

Noch haben wir keine langfristige Perspektive. Kurz vor der Einführung der DRG haben wir ebenfalls eine Umfrage durchgeführt. Da sind wir noch am Auswerten, was Unterschiede betrifft. Aber auch ungeachtet der DRG, die ja nur ein mögliches Anreizsystem unter anderen sind, wäre es absolut wünschenswert, ein umfassenderes, verbindliches Qualitätsmonitoring zu haben. Daten können sehr viel bewirken.

Haben Sie Anhaltspunkte dafür erhalten, dass Ärztinnen und Ärzte den Spagat zwischen

\section{medizinischen und ökonomischen Interessen} als problematisch empfinden?

Wir haben die Ärztinnen und Ärzte gefragt, wie sie sich auf einer Skala von 1 bis 10 gern einordnen würden, wenn man davon ausgeht, dass jeder Arzt sowohl das Patientenwohl berücksichtigt als auch die ökonomischen Interessen seines Spitals. 10 würde ausschliessliche Berücksichtigung der Patienteninteressen bedeuten, bei einer 1 würden nur ökonomische Interessen berücksichtigt. Die Befragten wünschten sich im

"Generell sollten Steuerungsgremien im Gesundheitswesen auf keinen Fall auf die ärztliche Expertise verzichten.»

Schnitt, bei 7,7 auf der Seite des Patienten zu sein, stuften sich in ihrem realen Arbeitsumfeld aber bei 5 ein. Ärztinnen und Ärzte handeln also ökonomischer, als sie dies eigentlich gerne täten.

\section{Wie bewerten Sie dieses Ergebnis?}

Zurzeit tut sich offenbar eine Schere zwischen Anspruch und Realität auf, was moralischen Stress oder Unzufriedenheit zur Folge haben kann. Interessant ist bei einem solchen Instrument aber auch die Beobachtung der Entwicklung über die Zeit. Vielleicht gibt es ja künftig Medizinergenerationen, die finden, dass «fifty-fifty» völlig okay ist und die beide Bereiche als gleichwertig ansehen. Mit solchen Fragen muss man sich gesellschaftlich auseinandersetzen.

Gibt es auch Chancen durch den ökonomischen Druck? Initiativen wie "Choosing wisely" oder "Smarter Medicine", die auf die Vermeidung nicht sinnvoller medizinischer Praktiken abzielen, lösen in breiten Kreisen eine positive Resonanz aus.

Je teurer das Gesundheitssystem wird, desto mehr wird man auf die Kosten schauen müssen. Das heisst, eine Ökonomisierung in diesem Sinne ist zunächst einmal unausweichlich. Dabei sollten Ärztinnen und Ärzte unbedingt eine aktive Rolle spielen, wenn es um Nachhaltigkeit und um die Vermeidung von Verschwendung geht.

Ärzte sollen also den Ökonomen und Gesundheitspolitikern sagen, wo ohne Qualitätseinbusse gespart werden kann?

Es ist sicher gut, dass Manager primär für die Kostenfrage und Ärzte primär für das Patientenwohl zuständig sind. Aber diese Arbeitsaufteilung darf nicht bedeuten, dass Ärzte nichts mit ökonomischen Fragen zu tun haben sollen. Denn dann kommt es zur Polarisierung, dann fehlt der Sachverstand und der Input der Ärzte, die beurteilen können, wo man etwas weg- 
lassen kann, ohne dass die Qualität leidet oder man damit dem Patienten sogar etwas Gutes tut. Werden solche Massnahmen von aussen dekretiert, ist das Resultat wesentlich schlechter, als wenn die Ärzte einbezogen werden.

\section{Wo und wie soll dieser Einbezug erfolgen? In politischen Gremien, auf Verwaltungsebene, bei den Krankenkassen, in den Spitalleitungen?}

Da gibt es unterschiedliche Ansatzpunkte. Bereits existierende Initiativen wie "Choosing wisely" sind sicher sehr sinnvoll. Im Bereich des Health Technology Assessments ist eine intensive Zusammenarbeit mit der Ärzteschaft nötig. Generell sollten Steuerungsgremien im Gesundheitswesen auf keinen Fall auf die ärztliche Expertise verzichten, wenn sie nachhaltig gute Resultate erreichen wollen. Auch ein verstärkter Einbezug der Medizinethik könnte helfen, eine einseitige Ausrichtung an Kennzahlen wie Patientenvolumen und Betriebsergebnis zu vermeiden.

\section{Zum Schluss noch ein Blick auf das grosse Ganze:} In welche Richtung muss sich unser Gesundheitssystem vor dem Hintergrund der aktuellen ökonomischen Herausforderungen entwickeln?

Ich glaube, dass nur ein dreifaches Ziel der Schlüssel sein kann, wie es im Konzept des sogenannten «Triple Aim» zum Ausdruck kommt. Wir wollen erstens eine gute individuelle Patientenversorgung. Zweitens wollen wir eine adäquate Versorgung der Bevölkerung, die die Schwerpunkte richtig setzt und die richtigen Probleme angeht. Aber drittens wollen wir auch, dass die Kosten nicht aus dem Ruder laufen und dass wir für das, was wir investieren, eine entsprechende Leistung bekommen. Ärztinnen und Ärzte sollen in diesem Gesundheitssystem keine faulen Kompromisse eingehen müssen, sondern eine am Patientenwohl

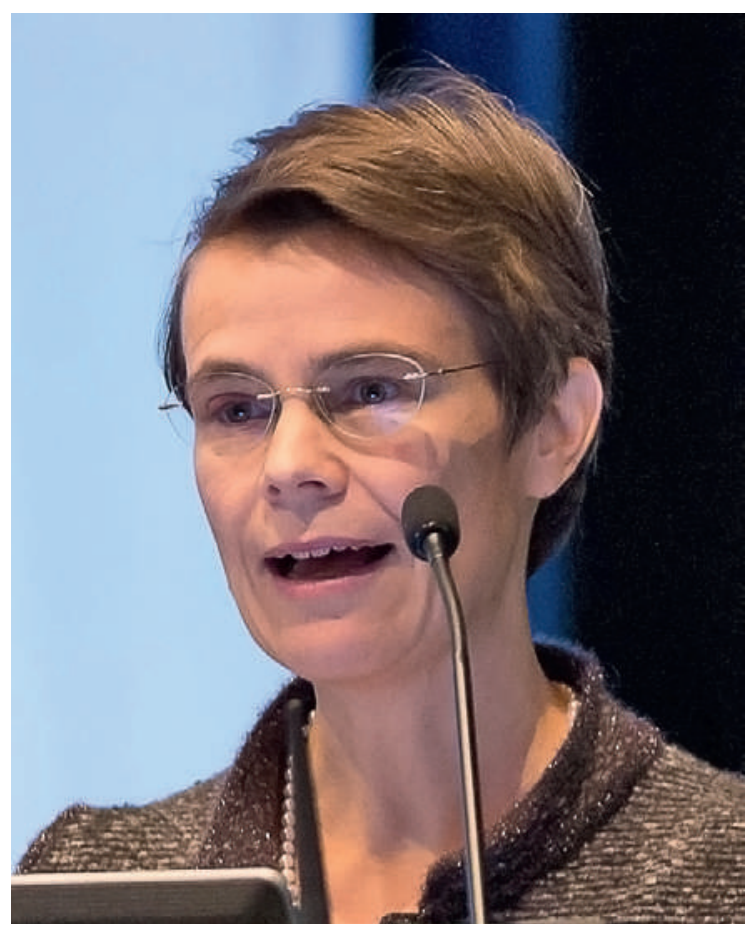

«Daten können viel bewirken»: Nikola Biller-Andorno plädiert für ein verbindliches Qualitätsmonitoring.

orientierte Medizin betreiben können, die auch die ökonomische Realität integriert.

\section{Referenzen}

1 Schweizerische Akademie der Medizinischen Wissenschaften Medizin und Ökonomie - wie weiter? Zusammenfassung und Empfehlungen des Positionspapiers der Schweizerischen Akademie der Medizinischen Wissenschaften. Schweiz Ärztezeitung. 2014;95(43):1598-16oo.

Das vollständige Positionspapier ist als PDF unter folgender Adresse im Internet zugänglich: www.samw.ch/de/Projekte/ Oekonomisierung-der-Medizin.html

2 Fässler M, Wild V, Clarinval C, Tschopp A, Faehnrich J, Biller-Andorno N. Impact of the DRG-based reimbursement system on patient care and professional practise: perspectives of Swiss hospital physicians. Swiss Med Wkly. 2015;145:w14080. 